\title{
KN-36
}

\section{Keynote Lecture}

Solving complex structures by electron crystallography: from inorganics to proteins

\author{
$\underline{\text { Xiaodong Zou }}^{1}$ \\ ${ }^{1}$ Department Of Materials And Environmental Chemistry, Stockholm University, Stockholm, Sweden \\ E-mail: xzou@mmk.su.se
}

Electron crystallography has several unique advantages in determination of complex crystal structures[1]. Nano- and micrometer-sized crystals that are too small for X-ray diffraction can be studied by electron crystallography. Structure determination by electron crystallography was first demonstrated using high resolution electron microscopy (HRTEM) imaging. Many complex structures including zeolites and quasicrystal approximants have been determined to atomic resolution by $3 D$ reconstruction of HRTEM images taken from different zone axes. A main drawback is that the technique requires high TEM and crystallographic skills and the structure determination is time-consuming. Recent developments in electron diffraction (ED) techniques have made large impacts in determination of complex structures, because of the development of automated ED data collection techniques. An important advantage of using electron diffraction is that the existing software packages for data processing and structure solution developed for X-ray diffraction are readily applied to ED data. In addition, electron dosages needed for electron diffraction is much lower than those needed for imaging, which is a great advantage for studying beam sensitive crystals such as metal-organic frameworks and proteins. A large number of complex structures, from zeolites, metal-organic frameworks, quasicrystal approximants, organic crystals to macromolecules have been determined from electron diffraction data (Figure 1).

Here recent developments of electron diffraction techniques, from automated diffraction tomography (ADT), rotation electron diffraction (RED)[2], MicroED/continuous rotation electron diffraction to serial crystallography using electron diffraction will be presented. The power of those techniques on the determination of complex structures from nano- and micro-sized crystals will be demonstrated. Using the RED data, it is not only possible to determine atomic positions in zeolites, but also to locate the sites with mixed occupancy (Si/B) and refine the occupancies. In combination with a hybrid pixel quantum area detector with high sensitivity and low background, the 3D data collection can be done in less than 20 seconds; the data quality is good enough to solve and refine the structure of a metal-organic framework to better than 0.10 $\AA$ in accuracy for all non-H atoms. Using dynamical refinement, more accurate atomic positions can be determined and even hydrogen atoms can be located, as demonstrated by Palatinus and co-workers[3]. Using a cryo-transfer sample holder, ED data can be collected from 3D nano- and micron-sized crystals of biomolecules and proteins embedded in amorphous ice and used for structure determination. Using serial crystallography recently developed in my group, it is possible to collect ED data from more than 500 crystals in less than an hour. This provides new possibilities for phase identification by electron diffraction, and for studying very beam sensitive crystals. We show that electron diffraction can be used as a stand-alone technique for structure determination of unknown crystals.

[1]X. Zou, S. Hovmöller \& P. Oleynikov, (2011) "Electron crystallography - Electron microscopy and electron diffraction", Oxford University Press.

[2] W. Wan, J. Sun, J. Su, S. Hovmöller, X. Zou (2013) J. App. Crystallogr. 46, 1863-1873.

[3] L. Palatinus, P. Brázda, P. Boullay,O. Perez, M. Klementová, S. Petit, V. Eigner, M. Zaarour, S. Mintova (2017) Science 355, 166-169.

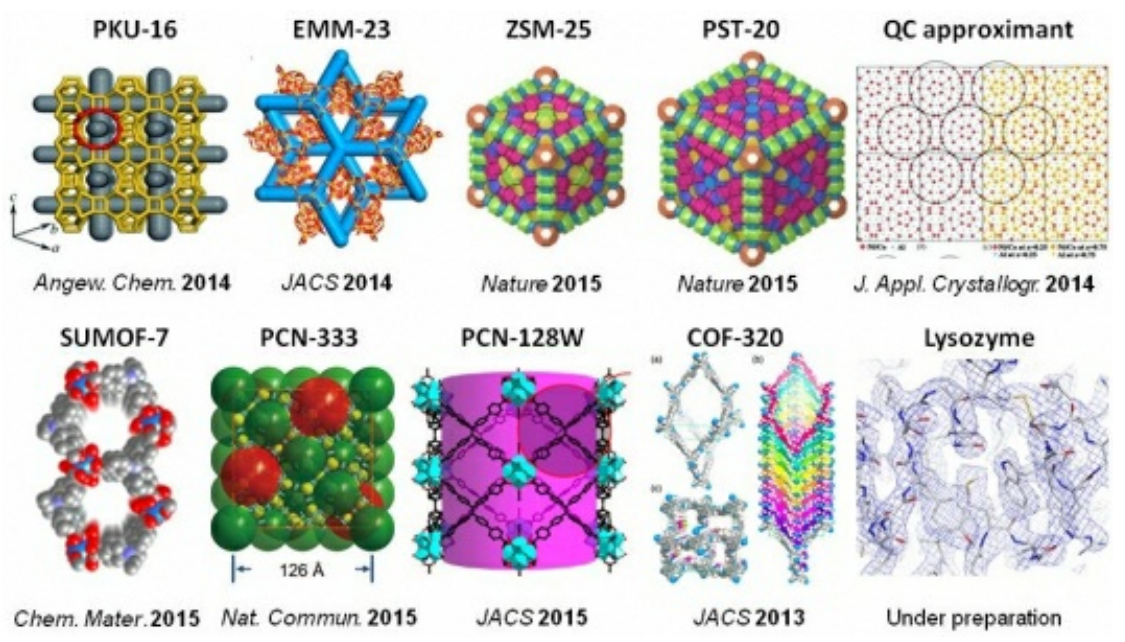

Keywords: Electron diffraction, structure determination, new methods 